\title{
Infertilidade associada a defeito microtubular dos espermatozóides de jumento (Equus asinus) avaliados por microscopia eletrônica de transmissão
}

\author{
Infertility associated with microtubular defect in a donkey (Equus asinus) spermatozoa evaluated by \\ transmission electron microscopy
}

\author{
André Maciel Crespilho ${ }^{1}$ Fernanda da Cruz Landim-Alvarenga ${ }^{2}$ Frederico Ozanam Papa ${ }^{2}$
}

RESUMO

Descreve-se um caso de infertilidade de um jumento SRD confirmada por meio de microscopia eletrônica de transmissão (MET). O espermiograma, avaliado sob microscopia ótica, revelou baixa motilidade e alta concentração de anormalidades espermáticas do tipo gota citoplasmática proximal. O material foi avaliado por MET, observando-se um acúmulo desordenado de microtúbulos causando protusões irregulares na região do colo espermático. O último teste realizado correspondeu ao de fertilidade in vivo, utilizando-se quatro éguas portadoras de bom histórico reprodutivo, nas quais não foi possível confirmar nenhuma prenhez. Frente aos resultados obtidos, associados aos achados da MET, estabeleceu-se o diagnóstico de infertilidade associada a defeito microtubular dos espermatozóides.

Palavras-chave: jumento, espermatozóides, defeito microtubular, microscopia eletrônica, infertilidade.

\section{ABSTRACT}

A donkey infertility was described by transmission electron microscopy (MET). The spermiogram evaluated by light microscopy showed low sperm motility and high concentration of abnormal sperm with proximal cytoplasmic droplets. The material was evaluated by MET, where it was observed disarrangement of microtubules, causing irregular protrusions in the spermatic neck. The last test done was the in vivo fertility, using four mares, with a reproductive healthy historic, where no pregnancy occurred. Facing the results that we had in vivo, associated with the MET findings, we diagnosed infertility associated with microtubular defect of the spermatozoa.

Key words: donkey, spermatozoa, microtubular defect, electron microscopy, infertility.

\section{INTRODUÇÃO}

Ao longo dos anos, muitos pesquisadores têm buscado desenvolver ensaios laboratoriais capazes de predizer com exatidão a capacidade fertilizante de amostras seminais (GRAHAM, 2001), permitindo dessa forma um manejo efetivo de reprodutores (KIRK et al., 2005). Nesse sentido, os espermatozóides são avaliados rotineiramente por meio de microscopia ótica, observando-se as características físicas relacionadas à morfologia e à qualidade do movimento desenvolvido pelas células espermáticas (JOSHI et al., 2001). No entanto, isoladamente, a morfologia e a motilidade espermática representam apenas indicações grosseiras da real capacidade fertilizante de um ejaculado (BLOTTNER et al., 2001), que depende, sobretudo, da integridade dos diferentes compartimentos celulares dos espermatozóides e de suas funções características (MAGISTRINI et al., 2001).

Embora os ensaios laboratoriais, como a determinação da motilidade espermática, possam ser utilizados para uma rápida avaliação de uma amostra seminal, esses testes não permitem o acesso às alterações que ocorrem em proporções sub-celulares (KIRK et al., 2005). Quando os defeitos morfológicos se expressam por alterações em escala nanométrica (nm), ocorrem falhas nos métodos tradicionais de avaliação, havendo a necessidade do emprego da microscopia eletrônica (JOSHI et al., 2001).

A ultra-estrutura dos espermatozóides de vertebrados e invertebrados tem sido intensivamente

\footnotetext{
${ }^{1}$ Programa de Pós-graduação em Reprodução Animal, Universidade Estadual Paulista Júlio de Mesquita Filho (FMVZ, UNESP). Distrito de Rubião Junior, s/n, 18610-000, Botucatu, SP, Brasil. Email: andremacc@yahoo.com.br. Autor para correspondência. ${ }^{2}$ Faculdade de Medicina Veterinária e Zootecnia (FMVZ-UNESP), Botucatu, SP, Brasil.
} 
estudada ao longo de muitos anos, especialmente por microscopia eletrônica de transmissão (ELLIS et al., 2002; JOSHI et al., 2001). A avaliação do sêmen eqüino por meio de microscopia eletrônica de transmissão (MET) é caracterizada pela observação de grande heterogeneidade morfológica e pela presença de várias anomalias que não podem ser evidenciadas pela microscopia ótica convencional (LANDIMALVARENGA\&ALVARENGA, 1997).

Descreve-se um quadro de infertilidade relacionada a asthenoteratozoospermia de um jumento SRD, apresentando uma alta incidência de espermatozóides portadores de gotas citoplasmáticas proximais à microscopia ótica.

Um jumento de 4,5 anos foi encaminhado ao Departamento de Reprodução Animal da FMVZUNESP, Botucatu/SP, em 15/04/2004, com um quadro sugestivo de infertilidade, uma vez que cobriu cerca de 50 fêmeas nas estações de monta de 2003 e 2004, não se confirmando nenhuma prenhez. O exame físico geral não revelou nenhuma anormalidade indicativa de enfermidade sistêmica. Ao exame específico externo, observou-se tamanho e consistência normal para ambos os testículos. A libido apresentava-se sem alterações, recebendo uma pontuação " 8 " (escala de 0 a 10).

Uma amostra seminal foi obitida por meio de vagina artificial ${ }^{\mathrm{a}}$, observando-se normalidade de volume e concentração espermática. No entanto, a análise computadorizada do movimento espermático, realizada por meio do aparelho Hamilton Thorn Research $^{\circledR}$, revelou baixa motilidade total $(\mathrm{MT}=20 \%) \mathrm{e}$ progressiva $(\mathrm{MP}=5 \%$ ), padrão que se repetiu em uma segunda coleta. A morfologia espermática foi determinada por meio de esfregaços corados pelo método de Karras modificado por PAPA et al (1988), observando-se 55,5\% de células normais; $18,5 \%$ de gotas citoplasmáticas proximais e $10,5 \%$ distais; $6,5 \%$ de anormalidades de peça intermediária, entre outros defeitos.

Com o objetivo de se determinar a origem primária da asthenoteratozoospermia, foram instituídas coletas seminais seriadas para realização de exames laboratoriais complementares. Em uma primeira análise, objetivou-se avaliar o efeito da retirada do plasma seminal e da adição de meios diluentes a base de leite em pó desnatado ${ }^{\mathrm{b}}\left(\right.$ Botu-Turbo $\left.^{\circledR}\right)$ sobre a motilidade espermática. Em uma segunda análise, foram avaliadas as características do sêmen frente à adição de plasma seminal heterólogo, testando-se a hipótese de imobilização espermática relacionada à produção de isoanticorpos anti-espermatozóides. Nesse teste, as células espermáticas foram separadas por meio de centrifugação ( $250 \mathrm{x}$ g por 10 minutos) e ressuspendidas em plasma seminal de um garanhão Árabe, portador de histórico reprodutivo normal.

A terceira análise correspondeu à preparação das amostras seminais para microscopia eletrônica de transmissão, segundo LANDIMALVARENGA\&ALVARENGA(1997). O ejaculado foi centrifugado a $300 \mathrm{x}$ g por 10 minutos, fixando-se o pellet formado em uma solução de glutaraldeído a 2,5\%, tamponado em PBS 0,1 $\mathrm{mol} \mathrm{l}^{-1}$, em pH de 7,4. Após 3 a 24 horas de fixação, os pellets foram enxaguados em solução tampão fosfato e pós-fixados em solução de tetróxido de ósmio a 1\%, tamponada em PBS, durante 3 horas à temperatura de $4^{\circ} \mathrm{C}$. Subseqüentemente, os espermatozóides foram desidratados em gradiente crescente de acetona $(30,50,70,90$ e $100 \%)$ por 5 minutos em cada escala. Foi utilizada resina Araldite 502 para as inclusões do material, que finalmente foi cortado (cortes ultrafinos de $50 \mathrm{~nm}$ ) e corado com solução saturada de acetato de uranila em álcool etílico $50 \%$ e, posteriormente, em citrato de chumbo. As amostras foram examinadas e fotografadas utilizandose o aparelho Phillips EM $^{\circledR} 301$.

A última análise correspondeu ao teste de fertilidade in vivo, realizado com 4 éguas de histórico reprodutivo sabidamente normal, acompanhadas ultrasonograficamente (Pie Medical Scanner ${ }^{\circledR} 200$ ) quanto ao desenvolvimento folicular a cada 12 horas. Todas as fêmeas tiveram ovulação induzida com 2500 UI de $\mathrm{hCG}^{\mathrm{c}}\left(\right.$ Vetecor $\left.^{\circledR}\right)$, procedendo-se as inseminações com 1 bilhão de espermatozóides totais, a cada 12 horas até a constatação da ovulação. Cada égua foi utilizada por 2 ciclos estrais consecutivos, sempre inseminadas com material proveniente do jumento em teste. O diagnóstico de gestação foi realizado com $12 \mathrm{e}$ confirmado aos 14 dias pós-inseminações por meio da ultra-sonografia trans-retal.

\section{RESULTADOS E DISCUSSÃO}

O primeiro teste indicou que a ressuspensão promoveu evidente melhora nas características seminais $(\mathrm{MT}=70 \%, \mathrm{MP}=46 \%)$, resultados provavelmente relacionados ao efeito estimulante da atividade flagelar proporcionada pelos açúcares que compõem o meio diluidor. Já a ressuspensão em plasma seminal heterólogo não revelou melhora apreciável nas características do ejaculado, descartando-se a possibilidade de toxicidade do tipo antígeno/anticorpos (MT=30\%; $\mathrm{MP}=12 \%$ ).

Ao exame de MET, foi observado um acúmulo desordenado de microtúbulos na região da 
cabeça e do colo espermáticos (Figuras $1 \mathrm{~A}$ e 1B). O significado desses microtúbulos não foi elucidado, porém sua ocorrência pode ocasionar protusões irregulares no colo, segundo LANDIM-ALVARENGA \& ALVARENGA (1997), semelhantes a gotas citoplasmáticas (Figura 1C), indicam uma anormalidade em alguma das etapas da espermatogênese, provavelmente de caráter genético. Originalmente, as gotas representam pequenas massas citoplasmáticas de 2-3 $\mu$ de diâmetro, sendo comumente encontradas em baixas porcentagens na região da cauda dos espermatozóides (BARTH \& OKO, 1989). No entanto, em estudos ultra-estruturais de espermatozóides eqüinos conduzidos por CACECI \& VARNER (1987), observou-se que em alguns casos muitos microtúbulos podem aparecer no interior das massas citoplasmáticas, sendo essa alteração classificada como defeito microtubular espermático, conforme relato anterior de LANDIM-ALVARENGA\&ALVARENGA(1997), que diagnosticaram a patologia em um garanhão Árabe, também portador de histórico reprodutivo de infertilidade.

Em relação ao possível caráter genético do defeito microtubular, ESCALIER (2003) indica que a enzima conjugada ubiquitina (UBE2B) está envolvida na organização das estruturas periaxonemais de ratos, observando-se, em animais "knock out", um grande prejuízo na espermatogênese em relação a falhas na morfogênese da cauda espermática. Nesse sentido, LATINI et al (2004) apontam uma clara importância de componentes genéticos nos quadros de infertilidade humana, relatando um caso de união consangüínea que resultou em um indivíduo do sexo masculino com
$100 \%$ de teratozoospermia, traduzida por agenesias de cauda espermática, defeitos microtubulares e alterações nas fibras que compõem o axonema. Uma possível explicação para estes achados corresponde à persistência da manchete espermática, representada por um conjunto de microtúbulos responsáveis pelo alongamento e formatação do núcleo das espermátides durante a espermiogênese (KNOBIL et al., 1994). A manchete não é normalmente observada em células maduras, evidenciando-se sua migração em sentido caudal durante a fase final do desenvolvimento das espermátides, formando um eixo de suporte para o canal flagelar (JOHNSON et al., 1997).

O defeito microtubular em espermatozóides de invertebrados também parece estar relacionado à persistência da manchete espermática, provavelmente como resultado do não-silenciamento de genes expressos exclusivamente durante a fase inicial da espermiogênese (BACCETTI et al., 2004). Nesse sentido, ESCALIER (2003) aponta o proteossoma 26S como componente protéico encontrado no interior da manchete, apresentando uma função marcadamente proteolítica durante a fase de remodelamento estrutural dos componentes da cauda espermática. Tendo em vista sua importância, torna-se plausível especular a não tradução do proteossoma $26 \mathrm{~S}$ como um possível fator causal envolvido nos quadros de defeitos microtubulares espermáticos.

Conclui-se então que a alta ocorrência de gotas citoplasmáticas observadas sob microscopia ótica relacionavam-se à presença de pequenas quantidades de citoplasma protruído, correspondente ao defeito microtubular observado, fator este

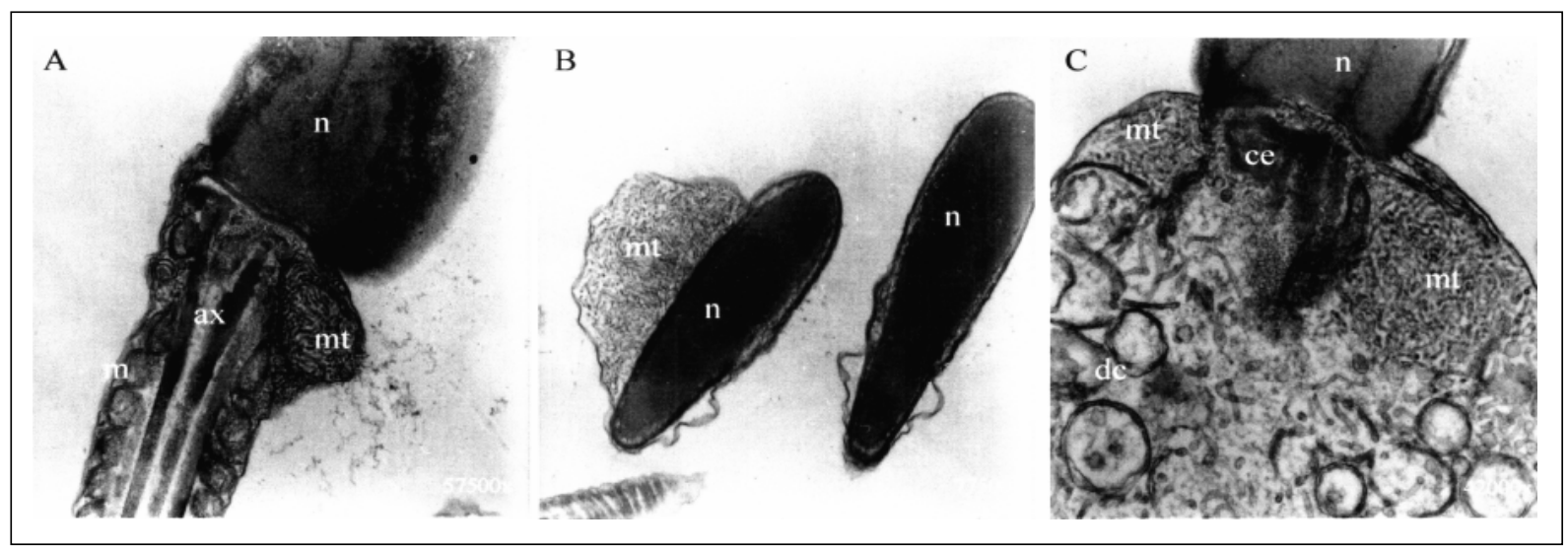

Figura 1 - Micrografias de espermatozóides de jumento obtidas por microscopia eletrônica de transmissão (MET). A) micrografia eletrônica mostrando o acúmulo de microtúbulos (mt) na região do colo espermático (ce); B) cortes transversais da região pós-acrossomal da cabeça espermática ilustrando o acúmulo de microtúbulos (mt) lateralmente ao núcleo (n); C) detalhe do colo espermático de espermatozóides com acúmulo de microtúbulos em ambos os lados; onde: ax= axonema; $\mathrm{m}=\mathrm{mitocôndria;}$ $\mathrm{dc}=$ debris celulares. 
determinante da baixa motilidade espermática póscoleta. Ao teste de fertilidade, não foi constatada nenhuma prenhez, resultados que, associados aos achados de MET, forneceram subsídios para o estabelecimento do diagnóstico definitivo do quadro de infertilidade do animal.

Em suma, a popularização das técnicas de MET na avaliação da infertilidade masculina humana e animal proporcionam a formação de uma base sólida para o melhor entendimento dos processos patofisiológicos envolvidos e para uma melhor adequação das condutas terapêuticas a serem seguidas. Até o presente, muitos aspectos relacionados à infertilidade do macho continuam obscuros, sobretudo nos quadros envolvendo componentes genético-familiares, linha de pesquisa carente por mais estudos, especialmente no tocante à expressão gênica diferencial da espermatogênese, base inicial dos diversos quadros de infertilidade.

\section{FONTES DE AQUISIÇÃO}

aVagina artificial e meio ${ }^{\mathrm{b} B o t u-T u r b o}{ }^{\circledR}$ (Biotech Ltda, Botucatu/ $\mathrm{SP})$.

${ }^{\mathrm{V}}$ Vetecor ${ }^{\circledR}$ (Laboratórios Calier do Brasil Ltda).

\section{REFERÊNCIAS}

BACCETTI, B. et al. An ultrastructural and immunocytochemical study of a rare genetic sperm tail defect that causes infertility in humans. Fertility and Sterility, v.82, n.2, p.463-468, 2004.

BARTH, A.D.; OKO, R.J. Abnormal morphology of bovine spermatozoa. Iowa: University, 1989. 285p.

BLOTTNER, S. et al. Morphological and functional changes of stallion spermatozoa after cryopreservation during breeding and non-breeding season. Animal Reproduction Science, v.65, p.75-87, 2001.

CACECI, T.; VARNER, D.D. Microtubular defects and persistent cytoplasmic droplets in an infertile stallion. The Southwestern Veterinary, v.38, p.13-16, 1987.
ELLIS, D.J. et al. Post-testicular development of a novel membrane substructure within the equatorial segment of ram, bull, boar and goat spermatozoa as viewed by atomic force microscopy. Journal of Structural Biology, v.138, p.187198, 2002.

ESCALIER, D. New insights into the assembly of the periaxonemal structures in mammalian spermatozoa. Biology of Reproduction, v.69, p.373-378, 2003.

GRAHAM, J.K. Assessment of sperm quality. In: INTERNATIONAL SYMPOSIUM ON STALLION REPRODUCTION, 3., 2001, Fort Collins, Colorado. Anais... Fort Collins: Colorado State University, 2001. 88p. p.23.

JOHNSON, L. et al. Factors affecting spermatogenesis in stallion. Theriogenology, v.48, p.1199-1216, 1997.

JOSHI, N. et al. Determination of the ultrastructural pathology of human sperm by atomic force microscopy. Fertility and Sterility, v.75, n.5, p.961-66, 2001.

KIRK, E.S. et al. Comparison of in vitro laboratory analyses with the fertility of cryopreserved stallion spermatozoa. Theriogenology, v.64, p.1422-1439, 2005.

KNOBIL, E. et al. The spermatozoon. In:_. The physiology of reproduction. 2.ed. New York: Raven, 1994. V.1, p.29-78.

LANDIM-ALVARENGA, F.C.; ALVARENGA, M.A. Microtubular defect in equine spermatozoa associated with infertility. Equine Veterinary Journal, v.29, n.6, p.487489, 1997.

LATINI, M. et al. Sperm tail agenesis in a case of consanguinity. Fertility and Sterility, v.81, n.6, p.1688-1691, 2004.

MAGISTRINI, M. et al. Assessment of sperm quality. In: INTERNATIONAL SYMPOSIUM ON STALLION REPRODUCTION, 3., 2001. Fort Collins, Colorado. Anais... Fort Collins: Colorado State University, 2001. 88p. p.24-25.

PAPA, F.O. et al. Coloração espermática segundo karras modificado pelo emprego do barbatimão (Stryphnodendrum barbatiman). Arquivo Brasileiro de Medicina Veterinária e Zootecnia, v.40, p.115-23, 1988. 